\title{
Designing a Mobile Diet Diary Application with and for Older Adults with AMD: A Case Study
}

\author{
Lilit Hakobyan \\ School of Engineering \& \\ Applied Science, \\ Aston University, \\ Birmingham, B4 7ET \\ hakobyl1@aston.ac.uk
}

\author{
Jo Lumsden \\ School of Engineering \& \\ Applied Science, \\ Aston University, \\ Birmingham, B4 7ET \\ j.lumsden@aston.ac.uk
}

\author{
Dympna O'Sullivan \\ School of Informatics, \\ City University London, \\ London, \\ EC1V OHB \\ dympna.o'sullivan.1@city.ac.uk
}

\author{
Hannah Bartlett \\ School of Life \& Health \\ Sciences, \\ Aston University, \\ Birmingham, B4 7ET \\ h.e.bartlett@aston.ac.uk
}

\begin{abstract}
Age-Related Macular Degeneration (AMD) is the UK's leading cause of severe visual impairment amongst the elderly. It accounts for 16,000 blind/partial sight registrations per year and is the leading cause of blindness among people aged 55 years and older in western countries (Bressler, 2004). Our research aims to design and develop a self-monitoring, ability-reactive technology (SMART) for users with AMD to support their dietary-based AMD risk mitigation and progression retardation over time. In this paper, we reflect on our experience of adapting and applying a participatory design (PD) approach to support the effective design of our application with and for older adults with AMD. We introduce the outcome of a series of PD sessions with older adults with AMD - that is, a paper prototype of our proposed application which focuses on accessibility for our target users - and discuss implications for the eventual prototype development.
\end{abstract}

UCD. Participatory design. Age-related macular degeneration. Older adults. Mobile assistive technology. Diet diary.

\section{INTRODUCTION}

With around 285 million people worldwide (WHO, 2013) and almost 2 million people in the UK (RNIB, 2013) living with sight loss, visual impairment is a serious age-related health concern among older adults (Congdon, et al., 2004). It has a significant impact on individuals' independence and quality of life, with $48 \%$ of visually-impaired people reported as feeling 'moderately' or 'completely' cut off from society (RNIB, 2013). The most common cause of sight loss in the UK is Age-Related Macular Degeneration (AMD); typically affecting people aged 50 and above, it impacts nearly one in ten of those over 80 (RNIB, 2013). As a progressive, degenerative disease of the eye it severely affects the macula, located at the centre of the retina, which is vital for clear central vision.

As highlighted by Figure 1, AMD presents a significant challenge in terms of user interface (UI) design - a challenge which is further complicated by the degenerative nature of the disease. Elderly people with AMD suffer from significantly limited independence and reduced quality of life (e.g., Mitchell, et al., 2008) as a result of the increased challenges associated with completion of daily activities. There is evidence that there is a link between dietary factors, AMD risk (Beatty et al.,
2001), and AMD progression (AREDS, 2001). The landmark Age Related Eye Disease Study (AREDS) showed that patients with high risk characteristics for AMD can lessen their risk of developing advanced AMD by taking appropriate nutritional supplements (AREDS, 2001).

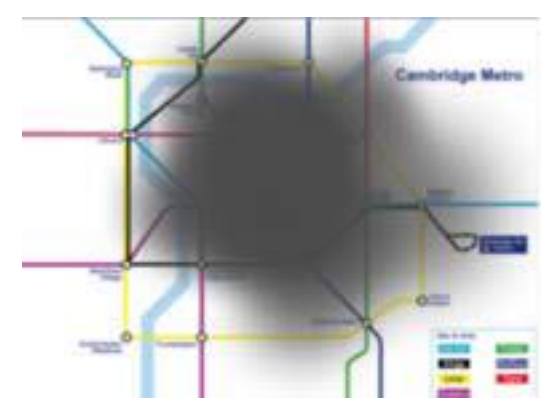

Figure 1: Example of how a subway map might be viewed through an eye affected by AMD [generated using www.inclusivedesigntoolkit.com].

Given the above, accurate and independent monitoring of diet, such that relevant personspecific dietary advice can be administered and behaviour change effected, is anticipated to be an important aid for persons with or at risk of AMD to maintain a healthy diet and therefore mitigate risk of developing and/or retard the progression of the disease. 
Electronic diet diaries have proven to be successful aids for improving independent living and care in fields such as diabetes for example (e.g., Tsang, et al., 2001); they have also been rated as a more effective way of collecting diary information (Stone, et al., 2003). Various electronic diet diaries are already available on mobile platforms - e.g., Fit4Life (Purpura, et al., 2011) - but fall short of identified needs for an AMD audience. They present visually-intensive Uls which are not adapted to people with visual impairment and do not support independent, accurate, and convenient use by users with AMD. Furthermore, existing systems do not make AMD-specific dietary recommendations that are customised for individual users with AMD based on their data or preferences combined with dietary best-practice.

We aim to address these known shortfalls by developing a mobile assistive healthcare application (SMART) to support accurate and convenient diet data collection on which basis to then provide customised dietary advice and recommendations in order to help support individuals with AMD to mitigate their ongoing risk and, ideally, retard the progression of the disease. This paper reports on our design activities conducted for the purpose of informing the development of SMART for older adults with AMD. Adopting a user-centred approach, we have commenced and will continue to include target users and stakeholders (e.g., persons with AMD, clinicians, carers, etc.) throughout all stages of our research, from the design to deployment of the SMART application, in an attempt to maximise the accessibility and acceptability of the application for people with AMD.

\section{BACKGROUND}

We recently surveyed advances in the field of mobile assistive technologies for the visually impaired and found evidence of innovation in the field of mobile assistive technology for the visually impaired that has the potential to substantively enhance the quality of life of visually impaired persons via, for instance, improved autonomy and safety (Hakobyan, et al., in press). Across the projects surveyed, however, we found little evidence of the direct involvement of target users. Where individual elements of UCD - including participatory design (PD), focus groups, and, most commonly, interview-based studies and user evaluation studies - had been adopted, they have demonstrated the extent to which they can assist designers in making informed choices in developing devices based on visually-impaired users' needs, wants and expectations. Although the use of user-centred design (UCD) methods is critical to the success of any technology development, we highlighted that this is especially true when entering and developing for a niche market, such as assistive technologies for the visually impaired.

A recent study examining the experiences and attitudes of older adults towards technology has found that (a) older adults are highly motivated to learn (or continue to learn) to use technology and (b), appropriate consideration of their lifestyles and the role of proposed technology is crucial to the design of such technology (Caprani, et al., 2012). While physical and sensory impairments associated with aging, and age itself, can essentially make technologies much harder to use (Hawthorn, 2000), the biggest limitation of technology use by the elderly is the fact that such technologies are not typically specifically designed to meet older adults' needs, wants and capabilities (e.g., Leonardi, et al., 2008). Advances in the field of gerontechnology (Bouma, et al., 2007) and approaches such as universal and inclusive design (Keates, et al., 2000) are encouraging designs that better consider the needs of users such as the elderly and the disabled.

Despite this, and the significant potential for mobile assistive technology to enhance visually-impaired people's healthcare and independent living, persons with AMD have not traditionally been directly involved in the design of technology to support their needs. They have been the focus of a study into handheld GUI-based computer interaction for older adults with AMD which identified that severity of the disease, design efforts and strategies, and contrast sensitivity were important indicators for successful iconic search using, and manipulation of, handheld computers by this user group (Leonard, et al., 2005). Beyond this, however, research into making technology accessible for this user group to date has been limited to a more general focus on desktop computers for the visually impaired rather than (mobile) assistive technologies for persons with AMD who, besides their challenges with focusintensive tasks (as a consequence of the degeneration in central vision), are also likely to suffer from other sensory and motor impairments (as a consequence of aging); as such, individuals with AMD find interaction with technology (and possibly participation in research studies overall) rather challenging.

\section{METHOD}

Following our commitment to use UCD methods for the design and development of our proposed application, and adopting the design process guidelines for designing for and with special needs user groups established by Leung et al. (2008), during the initial stages of our project, we: (1) 
consulted domain experts (e.g., clinicians, ophthalmologists) from several health organisations to attain an overview of AMD and the risk mitigation associated with dietary choices; (2) established contact with local community support groups for people with AMD and attended several of their meetings to allow us to build the relationships necessary to engender their trust and encourage their involvement in the PD stages of our research; and (3) conducted a serious of fieldwork activities that employed an integrated range of UCD methods, including focus groups and in-home observational studies with persons with AMD, for the purposes of knowledge elicitation (for detailed information about, and discussion of, our fieldwork activities see (Hakobyan, et al., 2012)).

One of the most significant realisations to emerge from our fieldwork activities (steps (2) and (3)) was the extent of observed heterogeneity of individuals' capabilities, meaning that their needs and, as such, acceptance of assistive technologies will vary substantially. We learned that, even for a given individual, technological needs will vary over time due to the degenerative nature of AMD. Our fieldwork activities highlighted that our application by virtue of being delivered on a mobile device and being independently accessible to and usable by persons with AMD - would have to facilitate customisation and personalisation of access based on its users' (varying) needs and capabilities. To allow us to continue (following on from alreadycompleted UCD methods) to fully embrace and be led by the complexities of our target users in our design practice, we decided to adopt the PICTIVE participatory design method for our research based on its central tenets of (a) the inclusion of end users as equal and valued members of the design team, and (b) the use of common office supplies rather than text documents or computer software as the design medium (Muller, 1992). We felt these underlying principles made the method well suited (with minor adaptations) to our user group and, since we anticipated that taking part in design work would be a new, and perhaps initially overwhelming task for our participants, we felt (and were encouraged by evidence from previous studies (e.g., Massimi, et al., 2007; Leung and Lumsden, 2008)) that the method had considerable scope to empower them to feel relaxed and able to contribute to the design work without the need for prior technical expertise or experience.

\subsection{Participants}

Having acquired a deep and relevant understanding of the needs, difficulties, and viewpoints of individuals with AMD via our previous UCD-based knowledge elicitation activities, we invited 4 already-involved individuals (out of 10 who were involved in our knowledge elicitation phases
(Hakobyan, et al., 2012)), comprising 3 with AMD and a carer who also had early stage AMD (see Table 1), to become integral members of our PD group; that is, they became full and active members of the design team for the purpose of hands-on design of our proposed technology to support their healthcare and independent living needs.

Table 1: PD participants' characteristics.

\begin{tabular}{|c|c|c|c|c|}
\hline ID & Age & Gender & $\begin{array}{c}\text { Experience } \\
\text { with } \\
\text { Computers }\end{array}$ & AMD Severity \\
\hline P1 & Mid 60s & Female & Moderate & Dry in 1 eye \\
\hline P2 & Late 70s & Female & None & $\begin{array}{c}\text { Dry/wet in } \\
\text { both eyes }\end{array}$ \\
\hline P3 & Late 80s & Female & None & $\begin{array}{c}\text { Dry/wet in } \\
\text { both eyes }\end{array}$ \\
\hline P4 & Late 80 s & Female & Some & Dry in 1 eye \\
\hline
\end{tabular}

Comprising members of different ages, different stages of visual impairment, and different levels of IT literacy, we believe our design team was representative of the heterogeneity of the AMD community whilst being ideally sized to encourage active participation (the number of participants in such studies in general and, in particular, in successful previous studies with vulnerable users are typically in this order (e.g., Leonard, et al., 2005; Massimi, et al., 2007)). We were delighted to have successfully recruited a team of this size, since finding and recruiting participants with special needs to such studies can be challenging (as reported in other research - e.g., Leung and Lumsden, 2008); this is particularly true of people with early diagnosis of AMD who often experience some degree of denial and who are not ready to self-identify and engage in support networks for AMD, placing them out of reach for studies of this nature. Whilst it could be argued that our lack of inclusion of individuals with early onset AMD might have led to skewed design objectives, we posit that the involvement of participants with advanced AMD enabled us to design the user interface such that it (a) can cope with worst case scenarios and therefore be used effectively and independently by persons with more advanced AMD which would not be the case if we had focussed on people with early onset AMD, and (b) will be adaptable to different levels of visual impairment over time, starting from the default layout of the UI which will be for nearer normal vision (and for which we did not require specialist input).

\subsection{Study Design and Logistics}

Over a period of 5 months, participants attended 8 design meetings in order to directly contribute, in an empowered way as experts in living with their condition, to the design of our SMART application. Given the potential issues of vulnerability associated with participants' capacity to read 
documentation associated with the study, in all phases of the study we paid particular attention to valid mechanisms for fully informing them about the work and obtaining their consent to participate. To this end, all documentation (including consent forms) was produced in various font sizes and distributed in advance of each phase of the study so that participants could turn to family/support workers to help them read the material and give them a chance to ask any questions before consenting to participate.

The design sessions took place at the university, in a room chosen for ease of access and good lighting. To remove physical participation barriers associated with commuting, return taxi-based transport was arranged and funded between participants' homes and the university. Participants actually commented that they enjoyed conducting this type of work within the university environment. To them, this reinforced the importance of the project and the significance of their contribution; it was regarded as highly motivational and made them feel 'very important' individuals.
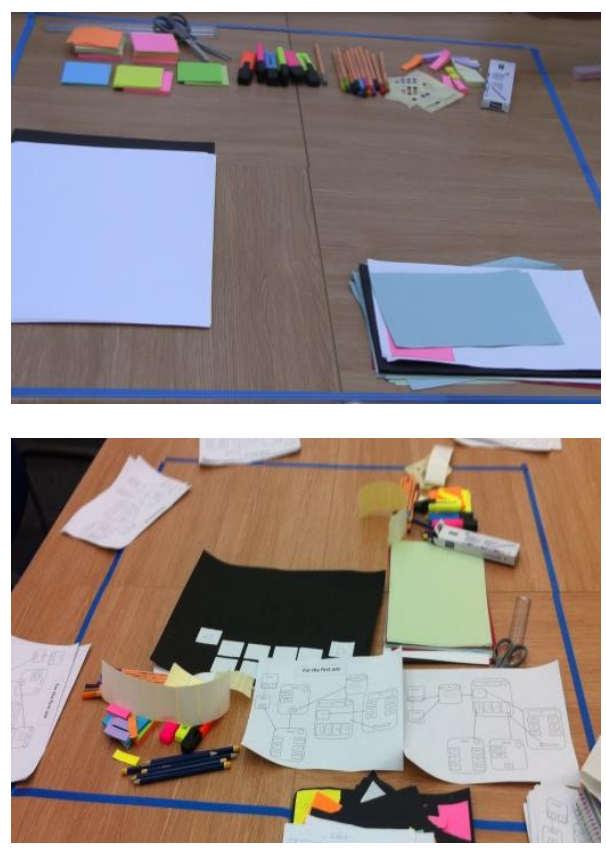

Figure 2: The shared workspace delineated in blue tape.

During the design sessions, participants were comfortably seated around a shared design surface on which they worked (see Figure 2). All sessions were recorded by a video camera (to which participants had consented); the area captured by the camera was delineated in blue tape (see Figure 2) on the design surface to ensure all relevant activities took place in view and to allow participants space to work 'off the record' if desired.

Since the PICTIVE PD process advocates that all participants should have equal stake and ownership of the process and the outcome, we opted for a relaxed structure to encourage participants to drive the process rather than being led through it. We had originally planned to constrain sessions to approximately 2 hours to avoid fatiguing our members but we encouraged participants to dictate the length of time they were willing to commit to each session, placing them in control. In reality, we found that participants were repeatedly deeply engaged in the process and always keen to continue their design work for longer, leading to sessions typically lasting 3-4 hours.

At the beginning of each session, the session goals were identified. Each session commenced with a summary of the previous session to recap the group's achievements as part of on-going encouragement given to participants and reinforcement of the value of their contribution and the fact that their healthcare and independent living goals are the driving force behind the design. As a concession to their visual impairment, rather than force participants to only view the central working version of the design, the researcher also created individual copies of the design for each participant to enable them to better and more comfortably view the content (often necessitating the design to be held at an angle next to their stronger eye - a viewing method which was uncomfortable if applied to the large, central copy of the design).

\section{DESIGN SESSIONS}

The first session was used to watch an explanatory video on the PD approach and to allow participants to ask questions which successfully relaxed them into the process. This was a very useful and practical method for introducing how PICTIVE sessions would proceed and for illustrating how simple office materials could be utilised to codesign paper prototypes. Additionally, we briefly explained to participants how these sessions allied with the overall scope of the project, what the subsequent stages would be and what the overall expected outcome was. We felt this to be essential for avoiding any mismatched expectations.

\subsection{Hardware Choice}

Despite advances in making mobile phones more accessible for the visually impaired, it was apparent from our knowledge elicitation activities and onoing discussions with our participants that such devices are not yet fully accessible or acceptable to people with AMD. In particular, the size of the display is a key limiting factor for this user group; the loss of central vision makes it very difficult to see fine details, especially important details placed in the centre of the display, with the result that they find the 'small' screens (and components contained therein) of mobile phones rather challenging. 
Consequently, we dismissed standard smartphones as our hardware choice, and from the first design session, agreed to focus on tablets instead. Participants identified some important features to be taken into consideration when selecting technology, namely it needs to:

- be lightweight and portable (hence our choice of mobile device);

- be easy to grip and hold (in particular for people with arthritis);

- be easily chargeable and to have reasonable battery life;

- $\quad$ support loud volume and vibrational output (in particular for people with hearing impairments);

- be hearing aid compatible; and

- support the taking of photographs (discussed in Section 4.2.2).

\subsection{Paper Prototyping}

Given the nature of our target audience, accessibility was at the heart of most design sessions. Initially, we began with a discussion on choice of colours, in particular the black/white foreground/background issue raised during our focus groups (Hakobyan, et al., 2012). We passed around both white and black paper onto which each participant could place some interface components (in the form of Post-It ${ }^{\mathrm{TM}}$ notes) in order to compare contrast effectiveness. It was apparent that the black background was most beneficial to those participants with the worst vision, whereas two participants with better eyesight (including the carer) preferred the white background. All participants (except the carer, who had early onset AMD) placed the components around the edges of the interface and left the centre clear; they also found 6-7 components per screen to be manageable (see Figure 3) - a key design finding that was only possible as a result of including users with late stage AMD. This activity afforded us an excellent and timely opportunity to expand on the prospect of personalisation - that is, how participants could individually tailor the application to better serve their needs and maximise application accessibility.

Since personalisation of the application was identified as a core requirement, we discussed, and participants strongly agreed to, the idea of presenting users with the option of completing automatic eye tests when they first open the application. This information would be used to personalise the interface to start with; thereafter; users would always have the option of manually 'altering' the settings. Following this, participants expressed the need for a brief instructions option on the first screen (in addition to manual instructions).
Having considered the requirements for launching or opening the application, we moved on to consider what was referred to by two participants who were slightly more computer literate as the 'home page' or 'main menu' for the application. Since participants found 6-7 icons per screen to be manageable, we limited the number of options per screen (including the main menu screen) accordingly, and this, to some extent, determined the overall system functionality.

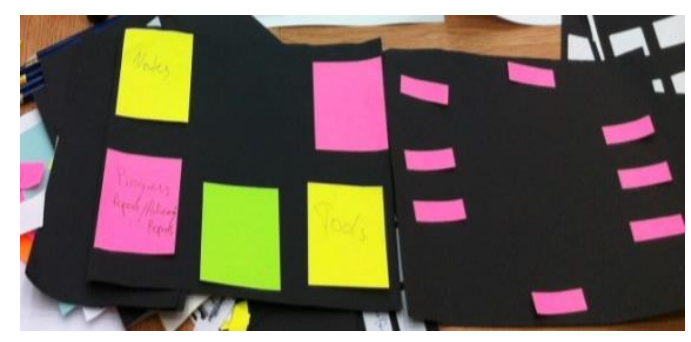

Figure 3: Participants' arrangements of the UI components.

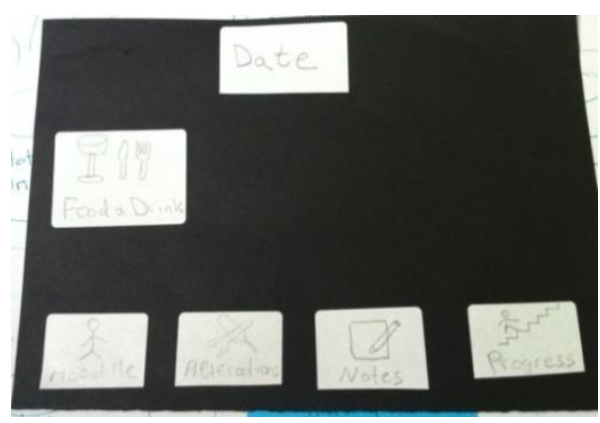

Figure 4a: The paper prototype of the 'Main Menu'.

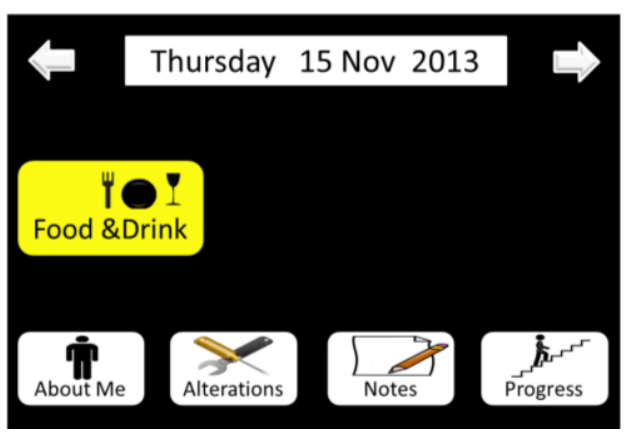

Figure 4b: Interactive mock-up of the 'Main Menu' created by the researcher using PowerPoint.

After much deliberation, participants agreed that the 'Main Menu' screen should comprise: (1) an option (later named 'Calendar') for viewing the calendar and selecting dates for food entry; (2) an option ('Progress') for viewing their progress made in terms of adherence to dietary recommendations, and for accessing their recommendations (it is important to note that users would be automatically provided with customised daily dietary advice); (3) an option ('Notes') to 'store' their 'ideas' and thus support their memory; (4) an option ('About Me') for recording a user's personal data (e.g., age, health 
condition, dislikes) for the purpose of providing customised dietary advice and recommendations; (5) an option ('Alterations') for altering or making changes to the screen and personalising it; and (6) as the key focus, an option ('Food \& Drink') for recording their daily intake of food. Together, we designed paper prototypes to reflect the main menu and subsequent screens (see Figure 4(a) for an example of the paper-based design of the 'Main Menu' screen), which the researcher later converted into higher-fidelity versions using PowerPoint (see Figure 4(b) for the higher-fidelity version of the 'Main Menu' screen).

\subsubsection{Icon Design and Command Naming}

A considerable amount of time during the PD sessions was assigned to discussion about icon designs, in particular to the advantages of their use (as opposed to reliance on words) in terms of immediate recognition (and, if necessary, recall) and increased application accessibility. Despite being unfamiliar with the whole concept of an icon and, as claimed by three participants, being unskilled in creative work, our participants were determined to design icons that were specifically accessible for their generation in contrast to those used in existing applications (as viewed on an iPad). Icon design actually proceeded hand-in-hand with decisions about the naming of the various functionalities/options within the application comprehensibility of existing functionality-related terminology on the iPad was also considered to be poor from our participants' perspective.

It is widely recognised that $\mathrm{UI}$ icons are not generally intuitive but are often learned. In accord with this, because our older adults have not been computer users throughout their lives, the existing icon designs and naming conventions used within current applications were not found to be consistent with our participants' mental models based on their life experiences, familiar environments and use of everyday objects; consequently, such icons served no useful purpose for them. For instance, the team vigorously debated the name for the 'Settings' and 'Tools' options on the iPad, arguing that these did not indicate to them they could alter or make changes to the screen and personalise the system. Initial alternative suggestions included 'Change my screen', but whilst participants believed it to be 'direct' they acknowledged it was 'unclear'. After much deliberation, our team decided to name the associated functionality within our application 'Alterations'. Our findings seem to be consistent with other research (e.g., Massimi, et al., 2007) which suggests that older adults' mental models do not match how current technology works, and more research on this needs to be undertaken to assist designers of future technologies. We deliberately did not pre-suppose the icon designs that would be useful to this audience and so the icon designs that emerged from our PD sessions encapsulated key design findings that would not have been uncovered without direct involvement of our users.

\subsubsection{Input Modalities}

An important (and challenging) aspect of designing the application was finding appropriate methods for inputting information into the application. One key example was the need to record their daily intake of food, with all participants agreeing that relying on text entry - either via touch or a stylus - to record this information would be extremely difficult for them. After some deliberation, we decided to mirror the 'eat well' plate approach recommended by the Department of Health (Department of Health, 2012).

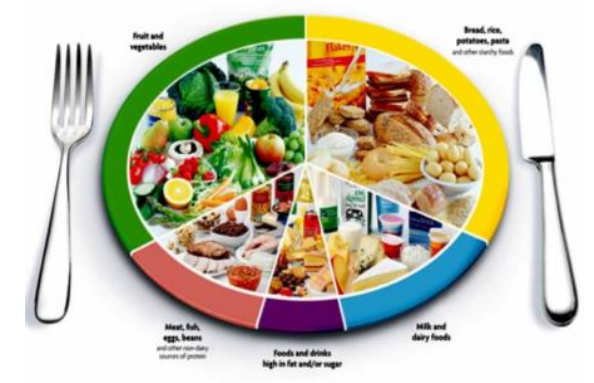

Figure 5: Eat-Well Plate

[http://www.dh.gov.uk/health/2012/06/about-the-eatwellplate/]

As illustrated in Figure 5, the plate provides a visual representation of the types and proportions of foods required for a well-balanced diet; in our case, based on similar visuals, users would select a food to enter by accessing the appropriate main food group and then selecting the specific food from that group. In the resulting application we plan to alter the type and number of food groups to keep the selection process simple and to focus detail (as opposed to higher-level food group selection) only where necessary to support the AMD-related dietary recommendations. For entering notes, participants similarly wished to avoid text-based data capture and instead expressed preference for taking pictures whenever possible. Having said that, we would always make an on-screen keyboard - for users to tap with a stylus or touch available as an alternative for those users who are capable of using it and who prefer a more direct input method. In this regard, users were unanimous in their stated preference for the use of a stylus over finger-based touch to input text via the keypad.

\subsubsection{Compliance - Motivational Mechanisms}

As a team, we explored motivational design features such as goal setting and virtual rewards as 
means to encourage engagement and proper use of the application. Motivation was seen by participants as linked to progress; they saw progress as being the main purpose of the application and, as such, a motivational factor in its use. This is illustrated in their inclusion of functionality for directly checking progress (the icon for which was designed to be a person climbing up stairs) - see Figure 4.

To further encourage and motivate users of the system to eat healthily and follow positive AMDrelated dietary recommendations, we explored the notion of goal setting as a motivational mechanism. To this end, we came up with the visual goalsetting concept of an interactive bar chart representing the most important vitamins/minerals (from an AMD health perspective) that would get filled up based on user's food intake. As a virtual reward (representing a further motivational mechanism) for following their daily food recommendations and advice, participants suggested providing users with extra hints and tips, including recipes for use of their favourite AMDbeneficial fruits and vegetables.

Interestingly, one participant suggested that comparing performance with friends could also be motivational. Due to time constraints we did not incorporate a specific functional feature to support this in our prototype design (recognising that the comparison can be, and may even be better, achieved outside of the technology itself) but plan to consider it further during the ongoing development of our application.

Together, we believe these recommendations stand as clear evidence of the progress participants were making in terms of understanding and envisaging technology and its use as a consequence of participation in the design process.

\section{PRELIMINARY EVALUATION}

During the last PD session, we briefly reviewed participants' thoughts on and experience of participating in the study process using a short questionnaire. The questionnaire consisted of a combination of five closed, 5-point scale questions (with $1=$ lowest and $5=$ highest scores) and a series of open-ended questions. Participants' overwhelmingly positive responses to the former are summarised in Table 2.

Our aim was to ask for participants' feedback on their experiences of participating in our research study and the UCD methods used (i.e., from focus groups to design sessions), and to assess their opinion of the end design of which they had stakeholder ownership. Whilst we acknowledge that our participants' responses could be argued to demonstrate clear evidence of the Hawthorne Effect, over the course of the research, the researcher and participants had developed mutual respect and a strong bond which allowed for open and honest exchange of ideas and opinions; as such, we hope that our findings are a true reflection of respondents' feelings.

Overall, participants' feedback indicated their delight and satisfaction in taking part in all aspects of the research study; they all also demonstrated a willingness (in fact keenness) to continue with the process. As anticipated, participants were similarly pleased and proud of the resulting prototype design; one participant thought it was simply 'excellent', and another one wrote:

'Others (i.e., people with $A M D$ ) will benefit from this design. I feel it is very simple to understand'.

In the last session, participants expressed disappointment that it was their last meeting for the time being. As one participant summarised:

'It was challenging, thought provoking and exciting! I would happily be involved in future projects of this nature. It has been a pleasure to be involved'.

Table 2: Average subjective scores (out of 5, with 5 being most positive) for closed post-design questions

\begin{tabular}{|l|c|c|c|}
\hline \multicolumn{1}{|c|}{ Question } & \multicolumn{3}{|c|}{$\begin{array}{c}\text { Average } \\
\text { Responses }\end{array}$} \\
\cline { 2 - 4 } & $\begin{array}{c}\text { Focus } \\
\text { Groups }\end{array}$ & $\begin{array}{c}\text { In- } \\
\text { Home } \\
\text { Studies }\end{array}$ & $\begin{array}{c}\text { Design } \\
\text { Sessions }\end{array}$ \\
\hline $\begin{array}{l}\text { Did you enjoy taking part in } \\
\text { this study? }\end{array}$ & 5 & 5 & 5 \\
\hline $\begin{array}{l}\text { Do you feel you have been } \\
\text { able to make a valuable } \\
\text { input to the study? }\end{array}$ & 5 & 5 & 5 \\
\hline $\begin{array}{l}\text { How useful do you think } \\
\text { each of the component parts } \\
\text { of the study were? }\end{array}$ & 5 & 5 & 5 \\
\hline $\begin{array}{l}\text { How easy did you find it to } \\
\text { participate in the various } \\
\text { sessions? }\end{array}$ & 5 & 5 & 5 \\
\hline
\end{tabular}

In order to understand how participants felt about their experience of the participatory design sessions with the benefit of reflection and hindsight, we conducted a one-to-one interview with 3 of our participants (unfortunately, our fourth participant could not take part due to ill health). Additionally, to further validate our prototype design, we attended a local community support group for people with AMD, during which six members of the group (none of whom had any prior involvement with our research) conducted a walkthrough of the interactive, higher-fidelity version of the prototype (see Figure 4(b)).

\subsection{Individual Interviews}


Individual interviews were conducted at participants' homes at their request, and occurred 3 months after the final PD session to encourage participants to comment with the benefit of hindsight. Each interview lasted about an hour: we summarise the findings here (for a comprehensive discussion of the findings from these issues see (Hakobyan, et al., 2013)).

The main objective of the interviews was to learn about participants' experiences of taking part in the PD sessions specifically, and their opinions about the manner in which the sessions were conducted (including their aim, the design space and pace of work). In particular, we hoped to determine what aspects they found interesting, valuable, confusing, challenging, etc., how similar sessions could be improved in the future, and to collect feedback on the actual prototype itself. All interviews were recorded and, subsequently, transcribed. All data collected was qualitative.

Overall, all participants very much enjoyed the design meetings. One participant's somewhat moving feedback aptly exemplifies and summarises all 3 participants' views on the design sessions:

\begin{abstract}
'It has given me an interest in what the blindness has done to me. So from those sessions it's a bit like seeing it rise from the ashes. It brought me a life, and for that I am grateful for, and, indeed, the friends that I made. It was fun, not only was it educational, it was fun! I looked forward, so it gave me an outlook of life really. It gave me something back that losing my sight had pinched away from me!'
\end{abstract}

Participants commented that the activities during the design sessions stimulated their learning, and that the PICTIVE PD sessions were a good way to learn about technology. Participants were 'surprised' to 'learn' and 'enjoy' something they previously did not understand:

'It came all together so wonderful, like a good play, brilliant, good plot, excellent!'.

Just as our participants learned about technology via their involvement, we learned a great deal about the needs, attitudes and perceptions of users with AMD, and older adults in general. Mutual learning throughout all stages was fundamental for the endurance and success of the project; we were reassured about participants' willingness to learn from and contribute to the PD sessions. Their positive interaction with computers (for those participants who owned computers) demonstrated that older adults (with AMD) could and would use technology if the potential benefits of such use are easily understood and appreciated.

As already mentioned, all participants expressed disappointment when the design sessions drew to a close and, remarkably, could not really offer any significant suggestions for improvements. They would have been happy to have continued with the process. They were all anxious to see "their" prototype implemented and put to practical use. In discussion, repeated reference to "their" design demonstrated the level of investment in and ownership they felt for the design. Participants' post-process feedback emphasised that participating in the project had heightened their perceptions of independence; they felt that they were effectively contributing to society (and their community) by being able to apply 'unrelated' skills (essentially their wealth of experience and knowledge) to the development of an assistive technology from which future generations could benefit. In general, themes of mutual learning, socialisation and empowerment emerged strongly from the feedback process. As such, we consider the process to have been a success - both methodologically for both parties, and in terms of the design output it generated.

\subsection{Validation of the Prototype Design}

Following the interviews, we attended a local community support group for people with AMD and asked volunteers - who had not previously been involved in any phase of the project - to provide informal, formative feedback on the higher-fidelity version of our prototype. The aim of this phase was to validate the prototype design in terms of its generalisability (i.e., intuitive command and option naming, icon design and placement of the user interface components) across more members of the intended user group The PowerPoint mock-up was shown on a laptop, but participants had also the opportunity to 'see' and 'experiment' with a Samsung tablet.

Three out of the 6 participants who volunteered said they would use the application. The other 3 were more reluctant to accept the concept of a dietary application, the underlying reason being scepticism that nutrition could have any positive influence on their eyesight at their later stage $(70+)$ of life. Interestingly, our design participants also recognised that the application was probably of little value to them given their stage of AMD progression, but were keen to identify means by which to help future generations avoid the vision loss they had endured.

Other than this, all 6 found the command/option naming and the design and use of icons to be 'clear', 'self-explanatory' and 'straight-to-the-point'. One participant explained that the 'clever' design and use of the icons would eliminate the need to read labels and, as such, make the interaction more 'enjoyable'. Furthermore, all agreed that the placement of the interface components was 
suitable, and offered no alternative arrangements. Participants did not actually comment on the hardware choice, but agreed that they would use similar devices as long as they could see the interface and its components.

\section{DISCUSSION}

Our work to date, whilst representing the design stages of an ongoing research agenda, has elicited valuable qualitative and previously unstudied information about the attitudes, needs, wants and capabilities of individuals with AMD as they relate to assistive technology and successful methods for its design. Based on our experience, we believe that direct, integrated participation of older adults with visual impairments (i.e., AMD) in the design process for assistive technology to support their needs (a) is essential in terms of teaching us about the potential positive impact that assistive technological solutions may have on their healthcare, independence and quality of life and (b), by virtue of supporting a deeper and more valid understanding of their needs, has the potential for significant influence on the success of technological development in terms of technology acceptance and ultimate impact.

At time of writing, we are about to commence the development of the application, to include the $\mathrm{UI}$ itself as well as the back-end which will involve the creation of an appropriate food ontology and the development of an intelligent computational engine that will combine information from the ontology with data from user profiles and dietary recordings from users to generate individualised recommendations to empower aging people with AMD to make informed dietary choices. Currently we are considering implementing these algorithms using recommender system techniques such as collaborative filtering or case-based reasoning.

We acknowledge that there are still many challenges to overcome before we arrive at the end product. The UCD process (including our paper prototype) has, however, highlighted some key features to be considered for and during the development of the end product. Firstly, the hardware choice is a deciding factor in the use (or abandonment) of technology by the AMD user group. The PD process has highlighted important features (see Section 4.1) that will need to be taken into consideration in any future hardware selection.

A major challenge identified in terms of the user interface design was the placement of the components such that it can be used effectively and independently by persons with AMD. This will require the atypical placement of the $\mathrm{UI}$ components around the edges, leaving the centre clear. Furthermore, we will have to develop adaptive algorithms to adjust the $\mathrm{UI}$ layout for a given individual based on auto-observed degeneration of visual acuity over time. To this end, given the importance of personalisation, we will investigate the use of artificial intelligence techniques for user profiling.

A further challenge will be the creation of appropriately abstracted food ontology. As noted, participants found inputting information rather challenging and, although our proposed 'Eat Well' plate concept should hopefully eliminate the need for 'typing', it will still require substantial visual attention in terms of reading available options (a challenge we must support effectively given the needs of our users). An important consideration for deployment of the application is its success at affecting behaviour change. Our design activities have highlighted participants' preferences for the practical motivational aspects associated with such change - e.g., our participants preferred charts and graphs for feedback purposes, and all agreed that beneficial feedback would motivate them to adhere to the dietary advice and recommendations. As already mentioned, we would also like to examine and evaluate one participant's suggestion that comparing performance with close friends would also be motivational. We intend to further examine users' viewpoints on whether to leave the comparison offline (i.e., during person-to-person discussion) or incorporate it into SMART, thus expanding the focus of our application from a userbased tool to a more "social" tool. Naturally, if the latter proves popular, we will need to consider, in close collaboration with users, the consequent privacy and security issues associated with such functionality provision. During our ongoing design and development activities, we intend to continue to include our end users in efforts to encapsulate manifestations of psychological models such as the Theory of Planned Behaviour (Ajzen, 1991) into the practical design to maximise the potential to support individuals with AMD in changing their dietary behaviour.

\section{CONCLUSIONS}

Working so closely with older adults with advanced disabilities, although sometimes challenging, has been highly rewarding and exciting. Our experience, combined with our participants' feedback, indicates that our tailored PICTIVE PD method was a valuable tool for involving users with AMD in design activities, and for encouraging them to act as fully empowered co-designers. Consequently, we have derived a user-led prototype design for an accessible mobile application to support older adults with AMD to make informed dietary choices. 
We intend continue with our commitment to applying UCD methods for the development and evaluation of our application, and will continue to involve target users directly throughout the process. We anticipate extensive evaluations of later implementations of our dietary application with an emphasis on: (1) accommodating the diverse and unique needs and capabilities of older adults with AMD; (2) UI personalisation based on their visual ability (or limitations); and (3) evaluating our proposed motivational mechanisms in terms of compliance and behaviour change in line with advice and recommendations.

\section{REFERENCES}

Ajzen, I. (1991). The theory of planned behavior. Organizational Behavior and Human Decision Processes, 50(2), 179-211.

Beatty, S., et al., (2001) Macular Pigment and Risk for Age-Related Macular Degeneration in Subjects from a Northern European Population. Investigative Ophthalmology and Visual Science, 42(2), 439446.

Bouma, H., et al., (2007) Gerontechnology in perspective: Review. Gerontech. Journal, 6(4), 190-216.

Bressler, N.M. (2004) Age-related macular degeneration is the leading cause of blindness. JAMA, 291, 1900-1901.

Caprani, N., et al., (2012) Technology use in everyday life: implications for designing for older users. In Proc. of the iHCl'12, Dundalk, Ireland, June $20-21,16-21$.

Congdon, N., et al., (2004) Causes and prevalence of visual impairment among adults in the United States. Archives of Ophthalmology,113, 477-485.

Crabtree, A., et al., (2003) Designing with care: Adapting cultural probes to inform design in sensitive settings. In Proc. OzCHI'03, Brisbane, Australia, Nov 26-28, 4-13.

Department of Health, (2012) Public health, adult social care, and the NHS: About the eatwell plate. http://www.dh.gov.uk/health/2012/06/about-theeatwell-plate/. Accessed Jan 25, 2013.

Hakobyan, L., et al., (accepted: in press), Mobile Assistive Technologies for the Visually Impaired, to appear in Survey of Ophthalmology.

Hakobyan L., et al., (2012) Understanding the ITrelated attitudes and needs of persons with agerelated macular degeneration: a case study. In Proc. of BCS-HCl'12, Birmingham, UK, Sept 12-14, 239-244.

Hakobyan L., et al., (2013) PICTIVE Participatory Design Process with Older Adults with AMD. In
Proc. of $\mathrm{iHCl}$ '13. Dundalk, Ireland, June 12-13 (in press).

Hawthorn, D. (2000) Possible implications of aging for interface designers. Interacting with Computers, 12, 507-528.

Keates, S., et al. (2000) Towards a practical inclusive design approach. In Proc. CUU'00, Arlington, USA, Nov 16-17, 45-52.

Leonard, V. K., et al. (2005) An exploratory investigation of handheld computer interaction for older adults with visual impairments. In Proc. of ACM SIGACCESS Conference on Computers and Accessibility. Baltimore, USA, 12-19.

Leonardi, C., et al. (2008) Designing a Familiar Technology for Elderly People. Gerontechnology, 7(2), 151.

Leung, R., \& Lumsden, J. (2008) Designing Mobile Technologies for Individuals With Disabilities, in Handbook of Research on User Interface Design and Evaluation for Mobile Technology, Information Science Reference, Hershey, USA, 609-623.

Massimi, M., et al., (2007) Using participatory activities with seniors to critique, build, and evaluate mobile phones. In Proc. of ASSETS'07, Arizona, USA, Oct 15-17, 155-162.

Mitchell, J., et al., (2008) The MacDQoL individualized measure of the impact of macular degeneration on quality of life: reliability and responsiveness. American journal of ophthalmology, 146(3), 447 -454.

Muller, M. (1992) Retrospective on a year of participatory design using the PICTIVE technique. In Proc. of CHI'92, June 3-7, 455-462.

Purpura, S., et al., (2011) Fit4Life:The Design of a Persuasive Technology Promoting Healthy Behavior and Ideal Weight. In Proc. of CHI'11, Vancouver, Canada, May 7-12, 423-432.

RNIB, (2012) Key Information and Statistics. http://www.rnib.org.uk/aboutus/Research/statistics/ Pages/statistics.aspx. (Accessed Mar 20, 2013).

Stone, A.A., et al., (2003) Patient compliance with paper and electronic diaries. Controlled clinical trials. 24(2), 182-199.

The AREDS Research Group, (2001) Archives of Ophthalmology, 119(10), 1417-1436.

Tsang, M.W., et al., (2001) Improvement in diabetes control with a monitoring system based on a hand-held, touch-screen electronic diary. $J$ Telemed Telecare, 7, 47-50.

WHO, (2013) Visual Impairment and Blindness: Fact Sheet No 282. http://www.who.int/mediacentre/factsheets/fs282/en /index.html. (Accessed March 20, 2013). 